\title{
Validation of the Physical Simulations of a Stirred Molten Metal Using Particle Image Velocimetry Data
}

\author{
AGNIESZKA DYBALSKA (1), ${ }^{1,2}$ DMITRY G. ESKIN,${ }^{1}$ \\ and JAYESH B. PATEL ${ }^{1}$ \\ 1.—BCAST, Brunel University London, Uxbridge UB8 3PH, UK. 2.—e-mail: dybalska.ag@gmail.com
}

Melt shearing refines the as-cast structure and the mechanism suggested beforehand is that of enhanced nucleation through oxide dispersion and fragmentation. The efficiency of processing by a rotor-stator mixer depends strongly on the treated volume of liquid metal. In this article the analytical modeling is validated by particle image velocimetry (PIV). The radius of the mixed region $(a)$ has been described as a linear function of rotational speed $(N)$ and the scale-up rules are given. Water model predictions have been confirmed experimentally by liquid metal shearing.

\section{INTRODUCTION}

The formation of oxide films in aluminum melts is unavoidable and harmful, as they facilitate porosity and cracking ${ }^{1-4}$ of the cast material. In recent years, instead of removing them from liquid metals, breaking large films and clusters into small fragments or particles has been suggested. ${ }^{5,6}$ It can be done through intensive melt shearing, using, for example, a rotor-stator device. Entrained oxides and films are usually distributed non-uniformly in the melt, and they are frequently large, folded and have poor wettability. ${ }^{1-3}$ The mechanical breakage of clusters and dispersion thereof in the liquid metal change the situation dramatically, since wetted and dispersed oxides may act as good nucleating substrates for aluminum and magnesium. ${ }^{7-10}$ A reduction in grain size as a result of melt shearing and the underlying mechanisms were reported elsewhere. ${ }^{11-14}$

The best processing conditions need, however, to be established. In this article, we aim at finding the dimensions of the well-mixed region (the so-called pseudo-cavern ${ }^{15}$ ) for aluminum alloys using a water model validated by particle image velocimetry (PIV) and by shearing of an aluminum alloy. The pseudocavern has been observed with Newtonian fluids by Ref. 15 and defined as a well-mixed region separated from the rest of a liquid by shear forces because of the fast flow of a fluid. According to Ref. 16, at low Reynolds numbers $(R e)$, the shape is that of a cylinder, and when $R e$ increases, the lower part of the pseudo-cavern grows more quickly than the upper part. From the pseudo-cavern images, the cross section can be approximated as an ellipse, as illustrated in Fig. 1 from references.

\section{MATERIALS AND METHODS}

To observe the flow patterns using tracing particles in a flow of liquid around a high-shear mixer (HSM), a physical model with water as a working fluid was used. As the essential properties of water at room temperature are similar to those of liquid aluminum and both are Newtonian fluids, water can mimic aluminum flow behavior and is often used as a modeling material (i.e., Refs. 19-22). The glass tank used had sizes $260 \mathrm{~mm} \times 260 \mathrm{~mm} \times 800 \mathrm{~mm}$ and the water level was kept at $200 \mathrm{~mm}$ from the bottom. On each occasion the HSM was placed in a slightly off-center position of the tank to avoid unnecessary surface vortexing in an unbaffled vessel. ${ }^{23}$ Still photos and movies were taken by a Fuji digital camera and a PIV system. ${ }^{24}$ The water was seeded with $8-12-\mu \mathrm{m}$ hollow glass spheres. ${ }^{25}$ A laser light sheet was placed just before the head of the HSM to avoid scattering of light from the metal impeller. With the laser operational, photograph sequences were taken at least 50 times in $100-\mu \mathrm{s}$ intervals (chosen experimentally) by the high-speed camera. With Insight-6 software, the vector and velocity magnitude plots were drawn with the expected maximum error of around 0.1 pixels. ${ }^{26}$ The HSM ("cross") used had a diameter of $21 \mathrm{~mm}$ 

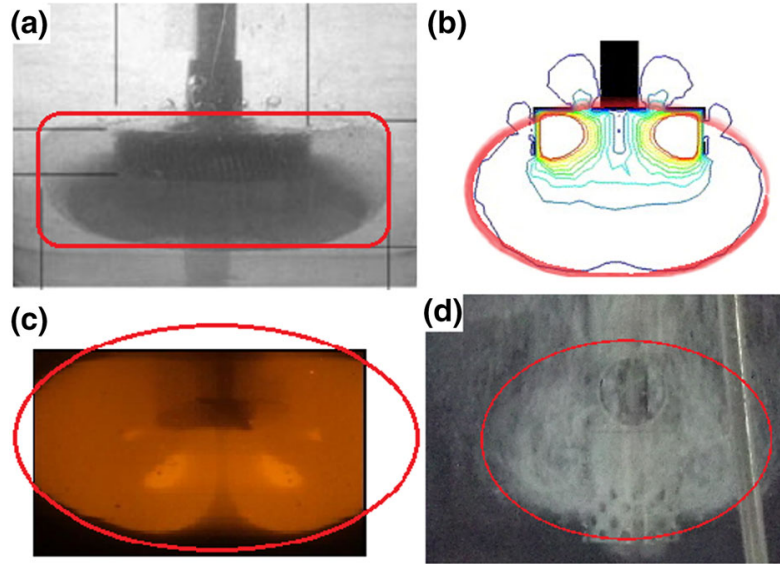

(d)

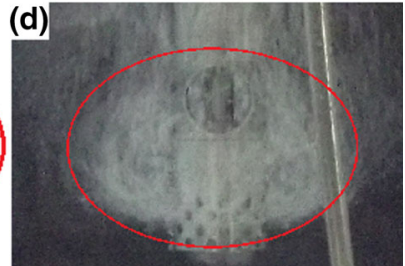

Fig. 1. The shapes of the well-mixed region (red cross section added for comparison): (a) assumed as cylindrical, small $R e$ (around 10) (adapted from Ref. 15); (b) elliptical cross section in a numerica model, $N=300$ (adapted from Ref. 16); (c) elliptical, around an open impeller (adapted from Ref. 17); and (d) elliptical, observed with gas dispersed in water by a rotor-stator mixer, $N=4000 \mathrm{rpm}, R e=$ 32,000 (reprinted from Ref. 18) (Color figure online).

with 48 round holes arranged uniformly in four rows on the stator, with a $15^{\circ}$ difference in hole positions between rows. The second head ("circle") differed in respect to the arrangement of the rows, which were parallel to the main axis of the impeller. The rotational speed $(N)$ of the HSM was controlled in the range of $2000-5000 \mathrm{rpm}$. To confirm the previous modeling, ${ }^{18}$ we have to keep the height above the bottom around $30 \mathrm{~mm}$ and that clearance results in scattered flow from the bottom. Thus, the flow before scattering has been analyzed. Both dimensions of the pseudo-cavern in directions $x$ and y were assigned the values $a$ and $H$ (the radius and height of the pseudo-cavern). Sizes of the pseudo-cavern were compared with those previously found by physical modeling. ${ }^{18}$

Pseudo-cavern sizes were measured from photographs obtained by the PIV system. In a typical HSM setup with a low clearance of the head from the tank bottom, some part of the flow was reflected from the bottom. The point at which jets of water were reflected from the bottom of the tank (or the jets were returned to the head if that happened earlier than reflection, as shown in the closer view in Fig. 2a) was one of the well-defined points of the ellipse (Fig. 2, point B), with the second point being set up on the upper line of holes on the HSM head where the jets began (Fig. 2, point A). The vertical axis of symmetry of the pseudo-cavern is the same as the main axis of the HSM head. From previous measurements in a water system, ${ }^{18}$ the ratio of both half-axes was known, equal to 1.35 , which allowed determining the shorter half-axis $(\mathrm{H})$. The tank side walls were far enough apart to avoid the jet scattering, and semi-major ellipse axis $a$ was measured from the drawn ellipses.
Further validation of water model experiments was carried out by melt shearing of an $\mathrm{Al}-3 \% \mathrm{Mg}$ binary alloy at $660^{\circ} \mathrm{C}$ under conditions determined using the validated water model with the optimal time of $180 \mathrm{s.}^{27}$ Following sample preparation, as described elsewhere ${ }^{27}$ the grain size was measured by a conventional linear intercept method for at least 50 grains. The standard error is calculated as the standard deviation of the Student $t$ distribution.

\section{RESULTS AND DISCUSSION}

\section{Pseudo-Cavern Radius}

Figure 2 shows overlays of the ellipses drawn onto PIV maps, demonstrating how we measured half-axis $a$ of the ellipse. The red outline shows the head position. In the sequence of photographs (two of which are shown in Fig. 2), we observed that the pseudo-cavern diameter increases with $N$.

Size $a$ found from these images was plotted against rotational speed $N$ and Re in Fig. 3. The linearization with the zero intercept of the data can be described as:

$$
a(N)=0.024 N
$$

The formula is valid for $N$ (rotational speed) given in rpm and $a$ in $\mathrm{mm}$.

From the presented data (Fig. 3) and previous work, ${ }^{18}$ this dependence can be used for both designs of the head (with crossed or straight hole lines). Additionally, similar results of measured size $a$ with both methods (PIV and modeling ${ }^{18}$ ) indicate that the chosen position of a laser sheet was close enough to exhibit nearly maximum values of velocities.

The $a(N)$ estimation, as described by Eq. 1, agreed well with previous estimates calculated from physical modeling. ${ }^{18}$ The linear dependence of the wellmixed region on $N$ can be related to the circulation, which occurs in the flow. Without being disturbed by the walls, the velocity of jets decreases while traveling from the head openings and the fluid returns to the head along the bottom of the tank because of the centrifugal action of the rotor, which acts as a pump. ${ }^{28,29}$ The movement of the fluid before reaching the bottom was approximately circular, and this motion can be described as a motion with a constant linear value of the speed (the resultant speed given by all forces present in the system). When the initial velocity increased, the jet length increased too, which meant that the radius of the circular loop was proportionally larger. These findings are also in good agreement with previous research by Ref. 30, who made PIV measurements of the flow caused by the rotor-stator batch mixer. The position of the PIV light sheet plane in Ref. 30 was perpendicular to the plane recorded in our work. Reference 30 focused on the flow around the stator holes and inside of the head. It was concluded in their work that "the calculated jet velocities and flow rates through the stator openings were found to 


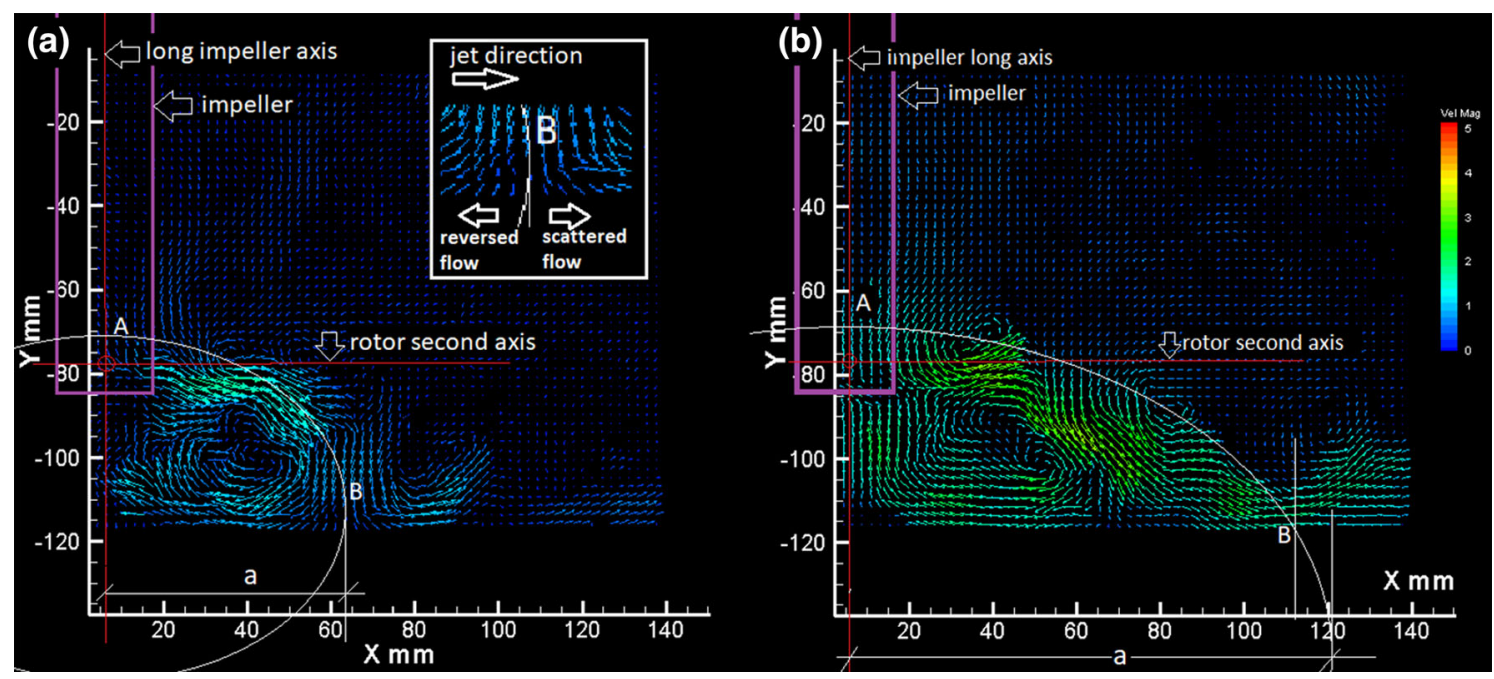

Fig. 2. Pseudo-cavern size a measurements for the "cross" head. Velocities on the scale bars are given in $\mathrm{m} / \mathrm{s}$. (a) $N=2000 \mathrm{rpm}$ and (b) $N=4000 \mathrm{rpm}$.
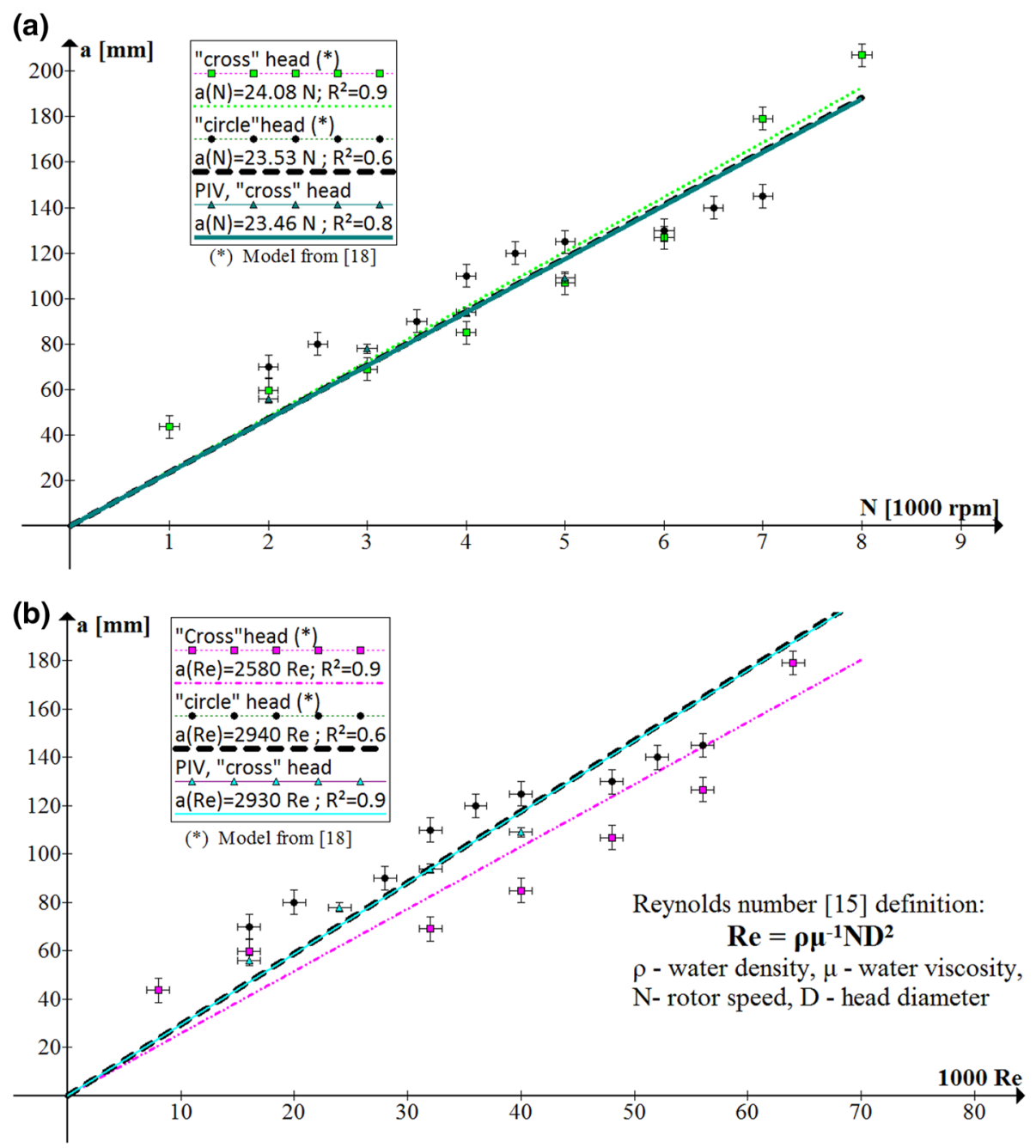

Fig. 3. Pseudo-cavern size a as the function of (a) $N$ and (b) $R e$ 
be proportional to the rotor speed while the energy dissipation rate scaled with the cube of rotor speed." Since the initial jet velocities were proportional to the rotor speed $(N)$, the pseudo-cavern size would be proportional to $N$, as found in this experiment.

\section{Scale-Up Rules}

Two main scale-up criteria of stirred tanks are widely used. The first is a constant tip speed criterion, and the second is a constant value of power per unit volume criterion. We chose the constant tip speed criterion because it was found to give more realistic predictions $^{31}$ applicable to the rotor-stator systems. ${ }^{30}$ Additionally, this criterion was based on the geometrical properties of the impeller, which were consistent with the approach used in the physical modeling described here, since viscosity was being treated as constant. To scale up the stirred system through the constant tip speed criteria, we have to compare the tip of the blade speed for each impeller compared. If we consider two identical impellers, except for the diameter, we can write the tip speed as:

$$
\begin{aligned}
& v_{1}=\pi d_{1} N_{1} \\
& v_{2}=\pi d_{2} N_{2}
\end{aligned}
$$

To describe two different scaling situations with the same tip speed, we can write:

$$
d_{1} N_{1}=d_{2} N_{2}
$$

Thus, if we scale up the rotor diameter to observe the same mixing, the rotational speed has to be decreased by the ratio of both impeller diameters:

$$
N_{2}=d_{1} d_{2}^{-1} N_{1}
$$

Considering the experimental model created in this work, we used the small head for modeling purposes, with rotor diameter $d_{1}=21 \mathrm{~mm}$. In a metallurgical laboratory, bigger impellers are commonly used, with diameter $d_{2}>d_{1}$. The model shows that the pseudo-cavern size depends on $N$, as in Eq. 1 . Taking Eq. 1 into account for the head with diameter $d_{2}$, we can write:

$$
a_{2}(N)=0.024 d_{1}^{-1} d_{2} N_{2}
$$

After using the constant value of $d_{1}=21 \mathrm{~mm}$, we can calculate:

$$
a_{2}(N)=1.14 \cdot 10^{-3} d_{2} N_{2}
$$

where $d_{2}$ is the diameter of a larger head than the one used in water modeling and $N_{2}$ is the rotational speed used in the new system, $a_{2}$ is the pseudocavern radius observed when head $d_{2}$ works with a rotational speed equal to $N_{2}$. All linear dimensions are given in $\mathrm{mm}$, and the rotational speed is in rpm.

Since both axes of the cross section of the pseudocavern are proportional $(a / H=1.35)$, the volume of the ellipsoidal pseudo-cavern can be calculated with

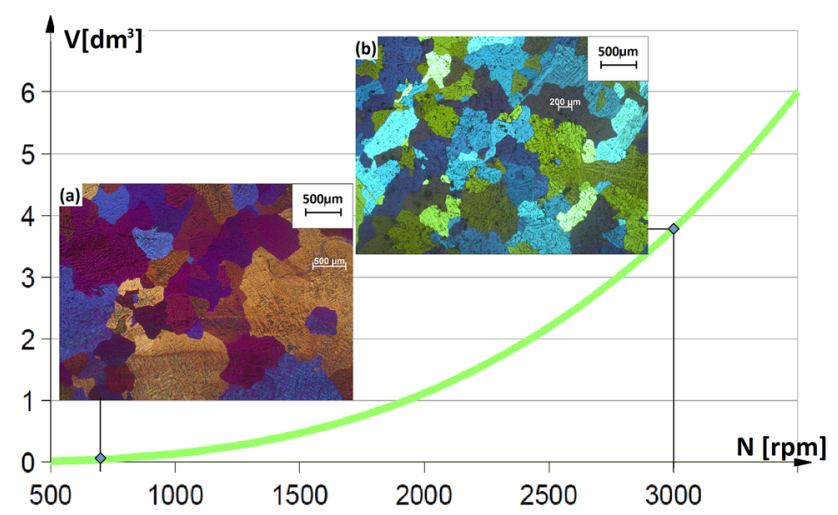

Fig. 4. Grain sizes of the Al- $3 \% \mathrm{Mg}$ alloy observed after $180 \mathrm{~s}$ shearing $\left(2.7 \mathrm{dm}^{3}\right.$ of liquid). (a) Without full agitation $(N=700 \mathrm{rpm})$ and (b) with full agitation ( $N=3000 \mathrm{rpm}$ ) overlaid on the graph of the agitated volume dependence on $N$ for the head used. ${ }^{18}$.

the known a described by Eq. 7. The detailed procedure of estimation of the volume according to size $a$ (as presented here) is described elsewhere. ${ }^{21}$

Using Eq. 7, we have calculated that the full mixing conditions for the crucible and the rotorstator mixer $(d=32 \mathrm{~mm})$ used for liquid metal experiments would be achieved at $3000 \mathrm{rpm}$; for comparison, we will use conditions with insufficient mixing, i.e., at $700 \mathrm{rpm}$.

\section{Proof of Concept with Liquid Metals}

The first conclusion from the model is a necessity to process liquid metal under the fully agitated conditions described by the model to avoid stagnant fluid zones wherein the potential oxide films cannot be processed. In this case, the number of dispersed oxides can be too small to act as a grain refiner, which results in larger grains. For verification of how the model predicts the efficiency of mixing with different $N$, a smaller rotational speed was chosen to cause insufficient mixing without full agitation, while a further speed increase resulted in full agitation. Figure 4 shows the expected volume of a pseudocavern with dependence on the used $N$ (calculated for the metal processing head, $d_{2}=32 \mathrm{~mm}$ ) and the microstructures obtained under chosen mixing conditions. The measured grain sizes after shearing without and with full-volume agitation were $512 \pm 35 \mu \mathrm{m}$ and $298 \pm 30 \mu \mathrm{m}$, respectively.

The average grain size after mixing under full mixing conditions decreased and was around 1.7 times smaller than those obtained under not fully agitated conditions. Experimental results showed good agreement with the physical model. Thus, the model can also be used in metallurgical applications for the selection of the best conditions.

\section{CONCLUDING REMARKS}

A model for predicting the mixing pseudo-cavern radius for liquid metal processing by a rotor-stator impeller was described as being dependent on the 
rotational speed of the impeller, $N$. Experimental data were collected using PIV techniques and compared with previous physical models ${ }^{18}$ with good agreement. The model can be used to estimate the sizes of well-mixed regions when the liquid has been processed by a similar impeller. The scale-up rules were used to make the model applicable to impeller sizes commonly used in metallurgy. The model predictions were checked experimentally by liquid metal shearing. Grain sizes in an aluminum alloy decreased after shearing under conditions described by the presented model.

\section{ACKNOWLEDGEMENTS}

Allocation of the equipment in the BCAST (Brunel University London) is highly appreciated. The first author is grateful for Ph.D. study funding from the Institute of Materials and Manufacturing, Brunel University London. The authors would also like to acknowledge Prof. Z. Fan, who initiated this research. The PIV measuring system was provided by the EPSRC Engineering Instrument Pool.

\section{OPEN ACCESS}

This article is distributed under the terms of the Creative Commons Attribution 4.0 International License (http://creativecommons.org/licenses/by/4.0/ ), which permits unrestricted use, distribution, and reproduction in any medium, provided you give appropriate credit to the original author(s) and the source, provide a link to the Creative Commons license, and indicate if changes were made.

\section{REFERENCES}

1. N.R. Green and J. Campbell, Mater. Sci. Eng. A A137, 261 (1993).

2. J. Mi, R.A. Harding, and J. Campbell, Met. Mat. Trans. A 35A, 2893 (2004).

3. C. Nyahumwa, N.R. Green, and J. Campbell, Trans. Am. Foundrym. Soc. V 106, 215 (1998).

4. Q.G. Wang, D. Apelian, and D.A. Lados, J. Light Met. 1, 73 (2001).

5. G. Scamans, H.T. Li, and Z. Fan, ICAA13, ed. H. Weiland, A. Rollett, and W. Cassada (Hoboken: Wiley, 2012), pp. 1395-1400.

6. H.T. Li, G. Scamans, and Z. Fan, Mater. Sci. Forum 765, 97 (2013).

7. Z. Fan, Solidification Science and Technology, ed. Z. Fan and I.C. Stone (London: Brunel University Press, 2011), pp. 29-44.

8. H. Men and Z. Fan, IOP Conf. Ser. Mater. Sci. Eng. 27, 012007 (2012).
9. S. Tzamtzis, H. Zhang, M. Xia, N. Hari Babu, and Z. Fan, Mater. Sci. Eng. A 528, 2664 (2011).

10. H.T. Li, Y. Wang, M. Xia, Y. Zuo, and Z. Fan, Solidification Science and Technology, ed. Z. Fan and I. Stone (London: Brunel University Press, 2011), pp. 93-110.

11. J. Patel, Y. Zuo, and Z. Fan, Proceedings of the 2013 International Symposium on Liquid Metal Processing and Casting, ed. M. Krane, A. Jardy, R. Williamson, and J. Beaman (Hoboken: Wiley, 2013),.

12. Y.B. Zuo, B. Jiang, Y. Zhang, and Z. Fan, IOP Conf. Ser. Mater. Sci. Eng. 27, 012043 (2012).

13. H.T. Li, Y. Wang, and Z. Fan, Acta Mater. 60, 1528 (2012).

14. H.T. Li, M. Xia, Y. Zuo, and Z. Fan, J. Cryst. Growth 314, 285 (2011).

15. L. Doucet, G. Ascanio, and P.A. Tanguy, Chem. Eng. Res. Des. 83, 1186 (2005).

16. F. Barailler, M. Heniche, and P.A. Tanguy, Chem. Eng. Sci. 61, 2888 (2006).

17. J.A. Sossa, Experimental and Computational Study of Mixing Behavior in Stirred Tanks Equipped with Side Entry Impellers (Vancouver: MASc thesis, University of British Columbia, 2007), p. 39.

18. A. Dybalska, Understanding and Development of High Shear Technology for Liquid Metals Processing (London: Ph.D. Thesis, Brunel University, 2016), pp. 71-96, 137207.

19. D. Gupta and A. Lahiri, Metall MaterTrans B 27B, 757 (1996).

20. D. Xu, W. Jones Jr., and J. Evans, Metall. Mater. Trans. B 29B, 1281 (1998).

21. L. Zhang, S. Taniguchi, and K. Matsumoto, Metall. Mater. Trans. B 38B, 63 (2007).

22. J. Karcz and J. Szoplik, Chem. Pap. 58, 9 (2004).

23. I. Tzanakis, G.S.B. Lebon, D.G. Eskin, and K.A. Pericleous, Ultrason. Sonochem. 34, 651 (2017).

24. M. Raffel, C. Willert, S. Wereley, and J. Kompenhans, Particle Imaging Velocimetry: A Practical Guide, 2nd ed. (Berlin: Springer, 2007), pp. 1-170.

25. TSI, Precision Measurement Instruments (Shoreview: TSI Incorporated, 2014).

26. H. Huang, D. Dabiri, and M. Gharib, Meas. Sci. Technol. 8, 1427 (1997).

27. A. Dybalska, D.G. Eskin, and J.B. Patel, JOM 69, 720 (2017).

28. A. Paipetis and V. Kostopoulos, Carbon Nanotube Enhanced Aerospace Composite Materials: A New Generation of Multifunctional Hybrid Structural Composites (Berlin: Springer, 2012), pp. 114-116.

29. J. Gülich, Centrifugal Pumps (Berlin: Springer, 2014), pp. 188-189.

30. H.H. Mortensen, R.V. Calabrese, F. Innings, and L. Rosendahl, Can. J. Chem. Eng. 89, 1076 (2011).

31. M. Edwards, N. Harnby, and J. Middleton, Fluid Mixing II. A Symposium Organised by the Yorkshire Branch and the Fluid Mixing Processes Subject Group of the Institution of Chemical Engineers and Held at Bradford University, 3-5 April 1984, 1st ed. (Oxford: Elsevier, 2013), pp. 107-126. 